\section{P-177 EVOLUTION AND REVOLUTION IN CARE HOMES; FRAILTY PROJECT ONE YEAR ON}

Jane Baumeister, Maddie Kinnar, Sue Phillipo. Garden House Hospice Care, Letchworth, UK

10.1136/bmjspcare-2019-HUKNC.199

Background Our geographical area was identified as having a higher proportion of care home beds than average which results in a disproportionately high workload for primary care. A report in 2008 (Joseph Rowntree Foundation) noted that patients in UK care homes received nearly twice as many GP contacts as similar aged patients in the community. The Frailty Community Nurse Specialist service was implemented in April 2018 and has been funded for two years to support GP workload in care homes.

Aims of the service

- To support primary care;

- To initiate advance care planning and escalation plans;

- To review residents within 48 hours of admission;

- To improve confidence of care home staff through education and therefore reduce primary care contacts;

- To provide triage for minor illness/signpost care home staff to alternatives to GP by use of flowchart.

Method Initial scoping exercise of care home admissions, education needs and end of life care planning undertaken to develop clearer understanding of needs of care homes, patients and GPs. The visibility of the CNSs has ensured that they are the point of contact for advice and input/signposting, liaison with relatives regarding advance care planning including DNACPR (Do Not Attempt Cardio-pulmonary Resuscitation) and treatment escalation plans.

Results Feedback from both GP practices and care homes has been positive with the service seeing over 1000 residents within the first year. This has significantly improved both patient, carer and family experience as patients are able to remain within care homes with the support of the Frailty CNSs for end of life care.

Conclusion Initial results from the first year of service demonstrate a positive impact on GP workload, a reduction in A\&E admissions and an increase in achievement of preferred place of care/death.

\section{P-178 WIDENING ACCESS TO HOSPICE CARE - SUPPORTING END OF LIFE CARE IN CARE HOMES}

${ }^{1}$ Helen Reeves, ${ }^{1}$ Katie Taroni, ${ }^{2}$ Joe Potts, ${ }^{2}$ Jeanette McCartney. ${ }^{1}$ St Giles Hospice, Whittington, UK: ${ }^{2}$ Douglas Macmillan Hospice, Blurton, UK

10.1136/bmispcare-2019-HUKNC.200

Background The national picture: the NSF for Older People emphasised the need to provide the right care in the right place at the right time. Hospital is not necessarily the best place for older people, unless they are in need of acute medical or surgical intervention (Department of Health, 2001). Admissions are expensive (Department of Health, 2004) and often a frightening experience, particularly to those who are frail and vulnerable; people with advanced dementia are particularly vulnerable and frequently admitted to hospital, often unnecessarily (Department of Health, 2009). In view of this, St Giles Hospice and Douglas Macmillan Hospice are working with South East Staffordshire + Seisdon Peninsula Clinical Commissioning Group to support care homes in providing end of life care to their residents by providing expert support and implementation of key tools to help identify residents in their last 12 months of life.

St Giles and Douglas Macmillan Hospice are supporting care homes throughout their localities by providing a specialist palliative care nurse to help support the home in identifying patients who may be in the last 12 months of life.

Aims and objectives of the service:

- Development of a 'Planning Ahead' register of frail patients at risk of admission or likely to be in the last 12 months of life, using recognised and evidence based prognostic indicator (s);

- To ensure residents on this register have a care plan and advance care plan where appropriate;

- To ensure resuscitation status is reviewed for patients and that support is provided for these discussions;

- To deliver reduction in the number of avoidable emergency admissions to hospital.

Outcomes and the future Currently St Giles Hospice and Douglas Macmillan Hospice are working with 46 homes providing weekly support sessions and review of the planning ahead register. Data is collated quarterly, with results due the beginning of July 2019.

\section{P-179 HOW CAN I LIVE WELL WHEN I'M DYING?}

Karen Tudge. Dorothy House Hospice Care, Winsley, UK

10.1136/bmjspcare-2019-HUKNC.201

Background The Live Well Partnership Coordinator (Band 4 Role) is an innovative and collaborative post. Run in partnership with Macmillan Cancer Support and the Royal United Hospital Bath, it brings together three organisations to identify and provide support and guidance to patients who are newly diagnosed with a metastatic condition or reoccurrence of their primary cancer. The post is based on the principles of supporting patients and families who are newly diagnosed with a metastatic illness and are 'living well'.

Previously the feedback demonstrated that this group of patients would have been seen by the acute teams for treatment and then sent home under the care of their GP. Feedback gained highlighted that patients and their families rarely sought support for anything related to their ability to live well and therefore any life enhancing and supportive services were not picked up until the problem became a crisis or the patient's condition deteriorated and they were referred to targeted palliative and hospice care.

Method and results Since the post started in May 2018 we have engaged in opportunities to highlight the importance of palliative care and how it can enhance and support life and not just support dying. Through working with; specialist nurses, palliative care teams, Emergency Department and medical teams we have been able to break down misconceptions about the role of hospice care and promote to professionals and patients the benefits of early engagement with hospice services, including:

- Information about planning ahead and advance care planning;

- Emotional support - re difficult conversations;

- Link with Dorothy House Community Services, e.g. Nurse led clinic, Coffee Club, COPE (Self-management course), carers support and young person's support; 